\title{
Strain Imaging of Arterial Wall with Reduction of Effects of Variation in Center Frequency of Ultrasonic RF Echo
}

\author{
Hideyuki Hasegawa $^{1,2}$ and Hiroshi Kanai ${ }^{2,1}$ \\ ${ }^{1}$ Graduate School of Biomedical Engineering, Tohoku University, Sendai, Japan \\ ${ }^{2}$ Graduate School of Engineering, Tohoku University, Sendai, Japan
}

\begin{abstract}
Atherosclerotic change of the arterial wall leads to a significant change in its elasticity. For assessment of elasticity, measurement of arterial wall deformation is required. For motion estimation, correlation techniques are widely used, and we developed a phase-sensitive correlation method, namely, the phased-tracking method, to measure the regional strain of the arterial wall due to the heartbeat. Although phasesensitive methods using demodulated complex signals require less computation in comparison with methods using the correlation between RF signals or iterative methods, the displacement estimated by such phase-sensitive methods are biased when the center frequency of the RF echo apparently varies. One of the reasons for the apparent change in the center frequency would be the interference of echoes from scatterers within the wall. In the present study, a method was introduced to reduce the influence of variation in the center frequencies of RF echoes on the estimation of the artery-wall strain when using the phase-sensitive correlation technique. The improvement in the strain estimation by the proposed method was validated using a phantom. The error from the theoretical strain profile and the standard deviation in strain estimated by the proposed method were $12.0 \%$ and $14.1 \%$, respectively, significantly smaller than those $(23.7 \%$ and $46.2 \%)$ obtained by the conventional phase-sensitive correlation method. Furthermore, in the preliminary in vitro experimental results, the strain distribution of the arterial wall well corresponded with pathology, i.e., the region with calcified tissue showed very small strain, and the region almost homogeneously composed of smooth muscle and collagen showed relatively larger strain and clear strain decay with respect to the radial distance from the lumen.
\end{abstract}

Keywords - ultrasound, center frequency, phase shift, radial strain of arterial wall, elasticity

\section{INTRODUCTION}

We have developed a phase-sensitive correlation method, namely, phased-tracking method, for estimating the displacement distribution in the arterial wall for strain imaging [1]. In such methods, ultrasonic pulses with finite frequency bandwidths are used. Therefore, the apparent change in center frequency occurs due to the interference of echoes from scatterers in the wall. Under such a condition, the displacement estimates are biased because it is obtained using the phase changes and center frequencies of received RF echoes. An autocorrelation-based method was proposed to compensate for this apparent change in center frequency $[2,3]$. In this method, center frequency distributions in two frames must be assumed to be the same. However, they become different when the scatterer distribution is changed by the artery-wall deformation.

In this study, a new method using the phase-sensitive correlation technique, which does not require the assumption that center frequency distributions in two different frames are the same, was examined for reducing the influence of center frequency variation on the estimation of artery-wall strain. The improvement of accuracy by the proposed method was validated using a cylindrical phantom. Furthermore, preliminary in vitro experimental results were obtained and are herein presented [4].

\section{METHODS}

\section{A. Displacement estimation using conventional autocorrelation methods}

The phases of echoes from a moving target, which is located at depth $d$ in the initial frame, are different in two consecutive $n$-th and $(n+1)$-th frames. This phase shift, $\Delta \theta_{d}(n)$, depends on the displacement of the target between these two frames. Therefore, the instantaneous displacement $\Delta x_{d}(n)$ between these two consecutive frames is estimated as follows:

$$
\Delta x_{d}(n)=\frac{c_{0} \Delta \theta_{d}(n)}{4 \pi f_{0 e}},
$$

where $c_{0}$ and $f_{0 e}$ are the speed of sound and the frequency used for displacement estimation, respectively. Phase shift $\Delta \theta_{d}(n)$ is estimated by the complex cross-correlation function $\gamma_{d, n}$ using the complex quadrature demodulated signals $z(d ; n)$ of received RF echo as follows:

$$
\Delta \theta_{d}(n)=\angle \gamma_{d, n}=\sum_{d \in R} z^{*}\left(d+x_{d}(n) ; n\right) \cdot z\left(d+x_{d}(n) ; n+1\right),
$$


where * shows the complex conjugate. The accumulated displacement $x_{d}(n)$ is obtained by accumulating the instantaneous displacement with respect to time.

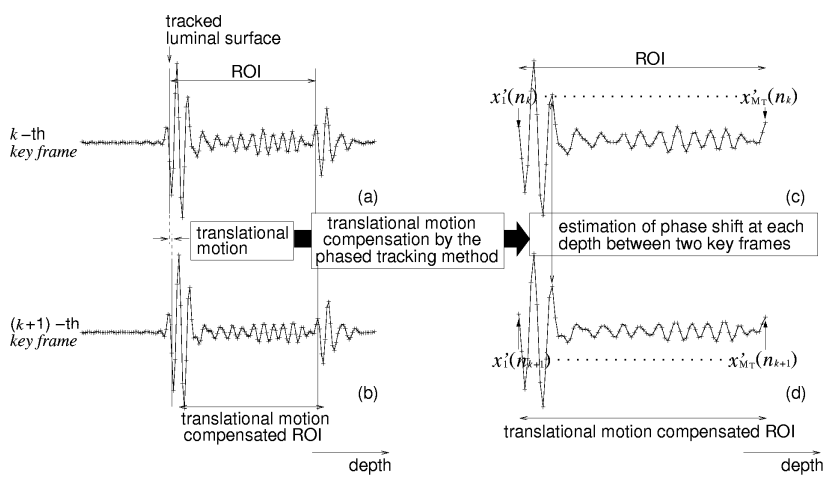

Fig. 1 Displacement estimation with translational motion compensation. Original RF signals in two consecutive (a) $k$-th and (b) $(k+1)$-th key frames. Translational motion compensated RF signals in (c) $k$-th and (d) $(k+1)$-th frames.

\section{B. Translational motion compensation}

The translational motion of the wall is compensated by tracking the echo from the luminal interface using the conventional method described in Sec. II-A because the echo from the luminal interface is dominant and is less influenced by the interference from other echoes from scatterers in the wall. In this study, key frames are defined as the frames at which the estimated displacement of the luminal interface $\Delta x_{d=l}(n)$ reaches the integral multiple of the spacing of the sampled points $\Delta X$. Figures $1(\mathrm{a})$ and 1(b) shows the RF echoes from the cylindrical phantom used in this study in two successive key frames. The region of interest (ROI) is manually assigned in the initial frame and, then, its position at each time is automatically determined by estimating the displacement of the luminal interface (the nearest edge of the ROI). The displacement of the luminal interface between two successive key frames is almost a spacing of sampled points, and the translational motion can be compensated by shifting the ROI by one point as shown in Figs. 1(c) and 1(d). After translational motion compensation, the phase shift $\Delta \theta_{d}(n)$, which is caused by the displacement contributing to strain, of echoes between these two key frames is estimated at each depth $d$ along the arterial radial direction, and the displacement distribution $\left\{x_{d}(n)\right\}$ is obtained from $\Delta \theta_{d}(n)$ using eq. (1). Finally, strain distribution is obtained by the spatial differentiation of displacements $\left\{x_{d}(n)\right\}$ in the arterial radial direction.

\section{Error correcting function}

After compensation of translational motion, the displacement contributing to strain is estimated using the phase shift of RF echoes. In such a situation, a large error resulting from the translational motion is removed, but error due to the mismatch between the frequency used for displacement estimation and the actual center frequency still remains in the estimation of the displacement contributing to strain even after the compensation of translational motion. In the present study, an error correcting function is introduced to further reduce this error. The error correcting function $\beta_{d, k}$ in $k$-th key frame ( $n_{k}$-th frame) is defined as follows:

$$
\begin{aligned}
\beta_{d, k} & =\frac{\frac{c_{0}}{4 \pi f_{0 e}}\left|\angle\left(\gamma_{d, k}^{\prime} \cdot \gamma_{d, k}^{*}\right)\right|}{\Delta X}, \\
\gamma_{d, k}^{\prime}= & \sum_{d \in R} z^{*}\left(d+x_{l}\left(n_{k}\right) ; n_{k}\right) \cdot z\left(d+x_{l}\left(n_{k}\right) ; n_{k+1}\right), \\
\gamma_{d, k}= & \sum_{d \in R} z^{*}\left(d+x_{l}\left(n_{k}\right) ; n_{k}\right) \cdot z\left(d+x_{l}\left(n_{k+1}\right) ; n_{k+1}\right),
\end{aligned}
$$

where $\Delta X$ is the spacing of sampled points. Key frames are assigned so as to be $\left|x_{l}\left(n_{k+1}\right)-x_{l}\left(n_{k}\right)\right|=\Delta X$, and the signal $z\left(d+x_{l}\left(n_{k}\right) ; n_{k+1}\right)$ in $\gamma_{d, k}$ is artificially displaced by a spacing of sampled points in comparison with $z\left(d+x_{l}\left(n_{k+1}\right) ; n_{k+1}\right)$ in $\gamma_{d, k}^{\prime}$. Based on eq. (1), this artificial displacement can be expressed as $c_{0} \mid \angle\left(\gamma_{d, k}{ }^{\prime} \gamma_{d, k}{ }^{*}\right) / 4 \pi f_{0}$ by the difference between angles $\angle \gamma_{d, k}^{\prime}$ and $\angle \gamma_{d, k}$ of $\gamma_{d, k}^{\prime}$ and $\gamma_{d, k}$, and it should be $\Delta X$. Equation (3) shows the ratio of the artificial displacement estimated by the phase shift $c_{0}\left|\angle\left(\gamma_{d, k}^{\prime} \cdot \gamma_{d, k}^{*}\right)\right| / 4 \pi f_{0}$ to actual one $\Delta X$. The error-corrected displacement $\Delta x_{\mathrm{c}, d}\left(n_{k}\right)$ between $n_{k}$-th and $n_{k+1}$-th key frames contributing to strain can be obtained as follows:

$\Delta x_{\mathrm{c}, d}\left(n_{k}\right)=\frac{1}{\beta_{d, k}} \frac{c_{0}}{4 \pi f_{0 e}} \angle \gamma_{d, k}$.

The displacement distribution is obtained by estimating the displacement $x_{\mathrm{c}, d}(n)$ at each depth $d$ which corresponds to the arterial radial direction. Finally, strain distribution is obtained by estimating the regional slope of the displacement distribution using the least-squares method [4].

\section{Experimental setup}

In this study, a cylindrical phantom and excised artery were measured by ultrasound. The outer and inner diameters of the phantom, made of silicone rubber (elastic modulus $E$ : $749 \mathrm{kPa}$ ), are 10 and $8 \mathrm{~mm}$, respectively. The phan- 
tom contains $5 \%$ carbon powder (by weight) to obtain sufficient scattering from the inside of the wall.

Figure 2 shows a schematic diagram of the measurement system. A change in pressure inside the phantom or artery was induced by circulating a fluid using a flow pump. The change in internal pressure was measured by a pressure sensor (NEC, Tokyo, 9E02-P16).

Pressure-diameter testing was applied to the phantom to obtain the elastic modulus of silicone rubber. In the pressure-diameter testing, the change in external diameter of the phantom was measured with a laser line gauge (VG-035, KEYENCE, Osaka, Japan).

In ultrasonic measurement, the phantom and artery were measured with a linear-type ultrasonic probe (Aloka, Tokyo, SSD-6500). The nominal center frequency was $10 \mathrm{MHz}$. RF echoes were acquired at $40 \mathrm{MHz}$ at a frame rate of $286 \mathrm{~Hz}$.

In the in vitro experiment, a femoral artery excised from a patient with arteriosclerosis obliterans was measured. The artery was placed in a water tank filled with $0.9 \%$ saline solution at room temperature. A needle was attached to the external surface of the posterior wall using strings to identify the section to be imaged during the ultrasonic measurement. After the ultrasonic measurement, the pathological image of the measured section was obtained by referring to the needle.

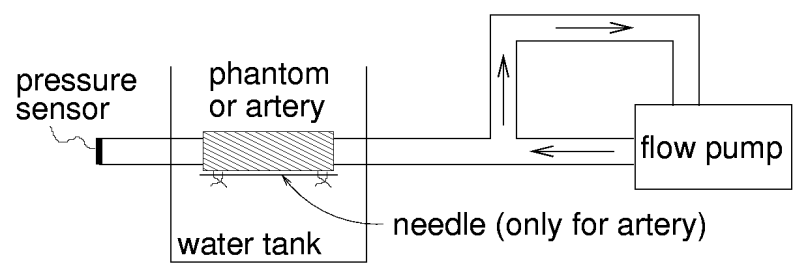

Fig. 2 Experimental system.

\section{BASIC EXPERIMENTAL RESULTS}

Figure 3(a) shows the strain distribution along the ultrasonic beam (the radial direction of the phantom) estimated by the conventional autocorrelation method [1]. Plots and vertical bars show means and standard deviations of 46 individual ultrasonic beams. The dashed line shows the theoretical strain profile, which is obtained by the measured internal pressure and elastic modulus $(E=749 \mathrm{kPa})$ of the wall measured by the pressure-diameter testing, given by [5]

$$
\varepsilon_{r}=-\frac{3}{2} \frac{r_{i}^{2} r_{o}^{2}}{\left(r_{o}^{2}-r_{i}^{2}\right) r} \frac{p}{E},
$$

where $r_{i}$ and $r_{o}$ are the original inner and outer radii, respectively.

Although mean values follow the theoretical strain profile, the standard deviations of strains estimated by the conventional method [1] are large. In Fig. 3(b), the center frequency estimation proposed in [2] was used together with the conventional method [1]. However, the strain estimates are not improved so much. Figure 3(c) shows the strain distribution obtained by the proposed method. Strain estimates were improved significantly by translational motion compensation and error correction.

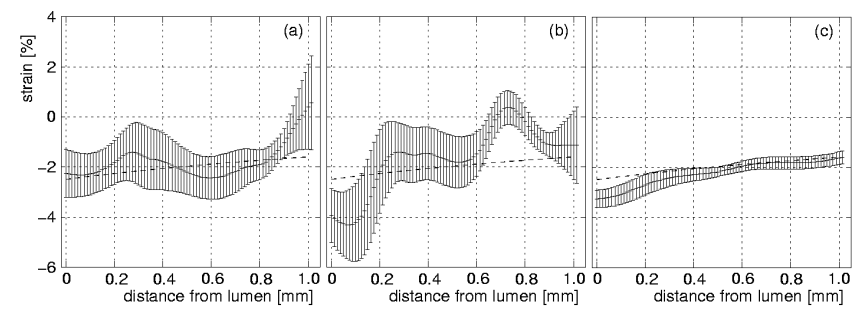

Fig. 3 Estimated strain distribution. (a) Conventional method [1]. (b) Conventional method with center frequency estimation [2]. (c) Proposed method with translational motion compensation and error correction.

\section{IN VITRO EXPERIMENTAL RESULTS}

Figure 4(a-1) and 4(b-1) show the B-mode images of two different regions in the excised femoral artery. The region shown in Fig. 4(a-2) shows very low strain. On the other hand, the region shown in Fig. 4(b-2) shows relatively larger strain, and there is a clear strain decay with respect to the distance from the lumen [5]. Figures 4(a-3) and 4(b-3) show the elasticity images obtained by eq. (8) using the measured internal pressure and estimated strain distributions. By comparing the elasticity images and pathological images of the corresponding sections shown in Figs. 4(a-4), 4(b-4), $4(a-5)$, and 4(b-5), the very hard region surrounded by the green line in Fig. 4(a-3) corresponds to calcified tissue. On the other hand, the region composed of smooth muscle and collagen shows a relatively homogeneous and lower elastic modulus distribution. These results show that the proposed method successfully reveals the difference in the deformation properties resulting from different tissue compositions.

\section{DISCUSSION}

The estimation of stress distribution in the arterial wall is one of the most difficult problems. In our study [6], the stress distribution in the arterial wall was assumed to be constant. Even in the case of a homogeneous cylindrical 
shell, the stress (strain) distribution is dependent on the distance from luminal surface. When an artery is a homogeneous cylindrical shell, the stress (strain) distribution can be theoretically derived based on eq. (8). However, it is difficult to estimate the stress (strain) distribution when an artery is inhomogeneous in the wall thickness and elastic properties. Therefore, in our study, the average stress was used to normalize the measured strain.
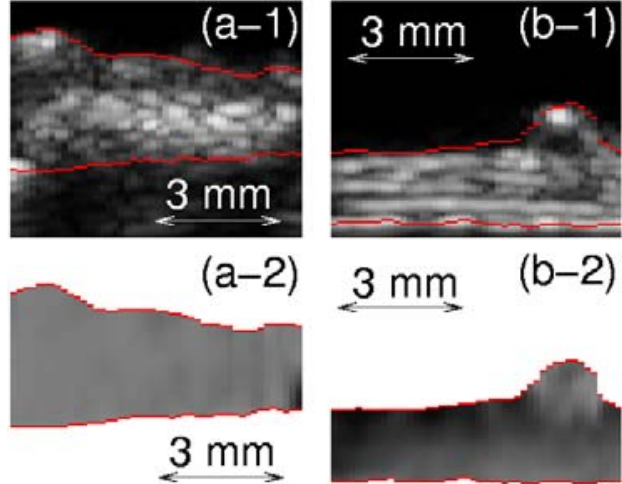

$(b-2)$
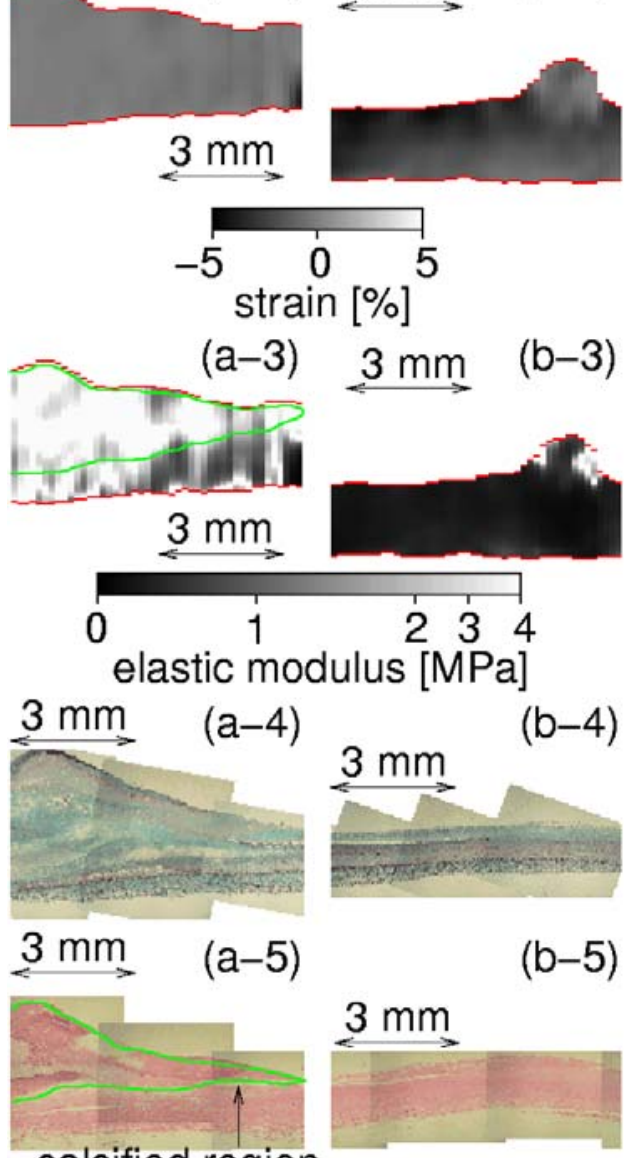

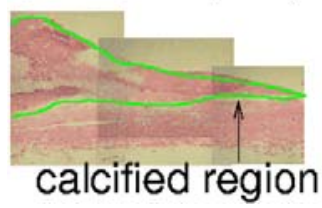

Fig. 4 In vitro experimental results for two regions in an excised femoral artery: (a) region with calcified tissue and (b) that without calcified tissue and composed of smooth muscle and collagen; (1) B-mode images, (2) strain images, (3) elasticity images, and (4) and (5) pathological images with elastica-Masson and hematoxilin-eosin staining, respectively.
This strategy is effective to compensate for the dependence of strain on the pulse pressure. When only the strain itself is considered to evaluate the elasticity, the strain is dependent on the pulse pressure which does not relate to the elasticity. As described in this paper, use of the stress distribution of a cylindrical shell given by eq. (8) is another solution to compensate for the pulse pressure variation.

\section{CONCLUSIONS}

In the displacement estimation based on the phase changes of echoes, the displacement estimates are biased when the center frequency of the RF echo changes. Such an apparent change in the center frequency could be caused by the interference of echoes from scatterers. To reduce the influence of the center frequency variation on the estimation of artery-wall strain, in this study, an error correcting function, which does not require the assumption that the center frequency distributions in two different frames are the same, was introduced. As a result, the proposed method provides better strain estimates in comparison with conventional phase-sensitive correlation methods.

\section{ACKNOWLEDGMENT}

We are grateful to Panasonic Co., Ltd. for providing the phantoms used in this study.

\section{REFERENCES}

1. Kanai H, Sato M, Chubachi N, et al. (1996) Transcutaneous measurement and spectrum analysis of heart wall vibrations. IEEE Trans Ultrason Ferroelect Freq Contr 43:791-810.

2. Loupas T, Powers JT, Gill RW (1995) An axial velocity estimator for ultrasound blood flow imaging based on a full evaluation of the Doppler equation by means of a two-dimensional autocorrelation approach. IEEE Trans Ultrason Ferroelect Freq Contr 42, 672-688.

3. Hasegawa H, Kanai H (2006) Improving accuracy in estimation of artery-wall displacement by referring to center frequency of RF echo. IEEE Trans Ultrason Ferroelect Freq Contr 53:52-63.

4. Hasegawa H, Kanai H (2008) Reduction of influence of variation in center frequencies of RF echoes on estimation of artery-wall strain. IEEE Trans Ultrason Ferroelect Freq Contr 55, 1921-1934.

5. Timoshenko SP, Goodier JN (1970) Theory of elasticity, 3rd ed., McGraw Hill, New York.

6. Hasegawa H, Kanai H, Hoshimiya N, et al. (2004) Evaluating the regional elastic modulus of a cylindrical shell with nonuniform wall thickness. J Med Ultrason 31, 81-90. 